\title{
Variability of Coronal Mass Ejections
}

\author{
Probhas Raychaudhuri \\ Department of Applied Mathematics, Calcutta University, Calcutta-700009, INDIA, \\ E-Mail: probhasprc@rediffmail.com
}

Coronal mass ejections (CME) from the solar corona are the most spectacular phenomena of solar activity. Solar physicists are tried to relate CME with other forms of solar activities. CMEs are the result of a large scale rearrangement of solar magnetic field and they are often observed as an eruption of twisted magnetic fields from the solar atmosphere. SOHO/LASCO detected (http://cdaw.gsfc.nasa.gov/CME_list) more than 7500 CMEs during 1996-2003 June. The catalog contains all the CMEs with primary characteristics e.g. linear speed, central position angle, and the angular width. We will use these characteristics to study the variations of CME within these periods. The period starts from the sunspot minimum to entire sunspot maximum range where the solar activity is high. Solar proton events $(E>10 \mathrm{MeV})$ were collected from NOAA website (http: /www.lep.gsfc.nasa.gov/waves) of the associated CMEs with halo CMEs. We find from CMEs data that the occurrence of average CME rate is 121.51 per month during June 1999 to June 2003 (sunspot maximum range) whereas the occurrence of average CME rate is 41.24 per month during January 1996 to May 1999 (sunspot minimum range), although during the year 1996 (when the average sunspot number is 8.6 per month) occurrence of average CME rate is 18.16 per month. The CME occurrence rate is also correlated with the sunspot numbers with high statistically significant level. The CME number is highest in 2002 but CME is higher in 2000 than in 2001. There is an overall similarity between sunspot number and CME rates but there are differences particularly from June 1999 which is the beginning of the sunspot maximum range. The CME rate peaks in September 2001 to October 2002, which is about 1.25 year after the sunspot maximum. Similarly the average speed of CME at the time of sunspot maximum range and sunspot minimum range are $575 \mathrm{~km} / \mathrm{sec}$. and $266 \mathrm{~km} / \mathrm{sec}$. respectively. This means that the average speed of CME increases from 1996 to June 2003. The CME speed is also correlated with the sunspot numbers with less significant level than the average rate of CME occurrence. The maximum monthly average speed is about $677.3 \mathrm{~km} / \mathrm{sec}$. at the time of April 2001, which is about 5 months earlier than the second sunspot maximum. From the preliminary list of halo CME events from SOHO/LASCO during January 1996 to June 2003 we find that the occurrence rate of average halo CME events during January 1996 to May 1999 is about 1.10 per month whereas during June 1999 to June 2003 is about 4.00 per month, during the year 1996 only two halo CMEs is occurred. We also find that the average speed of halo CME events during sunspot minimum range is $838 \mathrm{~km} / \mathrm{sec}$, whereas average speed of the halo CME events during sunspot maximum range is $1000 \mathrm{~km} / \mathrm{sec}$. Although during the year 1996 the average speed of halo CME events is $451 \mathrm{~km} / \mathrm{sec}$. From the characteristics of halo CMEs in years we find that the number of halo CME increases from 1996 to 2001 and the number of halo CME is maximum in the year 2001, after that number of halo CME decreases. In the 23rd solar cycle maximum solar activity occurred during June to September 2001 we call the time as 2 nd sunspot maximum time. We also find that number of high speed $(>1000 \mathrm{~km} / \mathrm{sec}$.) halo CME is highest during 2nd sunspot maximum range (i.e., during 2001-2002). We find from the halo CME data that average halo CME speed increases from 1996 to 1998 and then decreases from 1998 to 2000 and again increases from 2000 to 2003 and we expect that the average speed of halo CME will decrease after 2003. We find 78 solar proton events $(E>10 \mathrm{MeV})$ from CME and about 43 of them are from halo CME during 1996 to 2003. We noticed that the maximum solar proton events occurred at the second sunspot maximum, which is occurred after $1 \frac{1}{2}$ sunspot maximum in the 23rd solar cycle. We find there exist 5 phases of solar proton events $(E>10 \mathrm{MeV})$ data in the 23rd solar cycle. The first phase is at the sunspot minimum, 2nd phase is after two years from the sunspot minimum, 3rd phase is at the time of sunspot maximum 
and 4th phase occurs just one and half year (usually it is about $2 / 3$ years) after the sunspot maximum and 5 th phase occurs $2 / 3$ years before the sunspot minimum. We find six solar proton events $(E>10 \mathrm{MeV})$ data within 1999 to 2003 with 12900 to 31700 pfu which produced strong geomagnetic storms and all of them are very high-speed halo CME. It is known that very fast CMEs $\left(V_{p}>1000 \mathrm{~km} / \mathrm{sec}\right.$.) are capable of causing extremely intensive geomagnetic storm when $D_{s t}$ index $<-300 n T$. We find that there is a significant correlation between the speed of the $\mathrm{CME}$ and solar proton events $(E>10 \mathrm{MeV})$ data. Solar radius measurement at Rio de Janeiro from 1997-2000 shows that the solar radius varies in phase with the solar cycle. Astrolabes of Antalya, Rio de Janeiro and Santiago suggest that the solar radius varies in phase with the solar cycle. From the detection of solar radius variations with MDI on board SOHO it is found that the solar radius increases with the number of sunspots[1]. It appears that solar radius variation and solar neutrino flux variation with the solar cycle is due to the variation of solar core pulsations and is mainly responsible for the variation of CME and its speed that is in phase with the solar cycle. We suggest that the above-mentioned characteristics are interrelated and that a pulsating solar core may be their common origin [2].

Keywords. Sun: activity, coronal mass ejections (CMEs), magnetic fields, particle emission

\section{References}

Noel, F. 2002, Astronomy and Astrophysics 396, 667

Raychaudhuri, P. 1994, Solar Phys. 153, 445. 